C-A/AP/\#413

Dec 2010

\title{
Copper Coating Specification for the RHIC Arcs
}

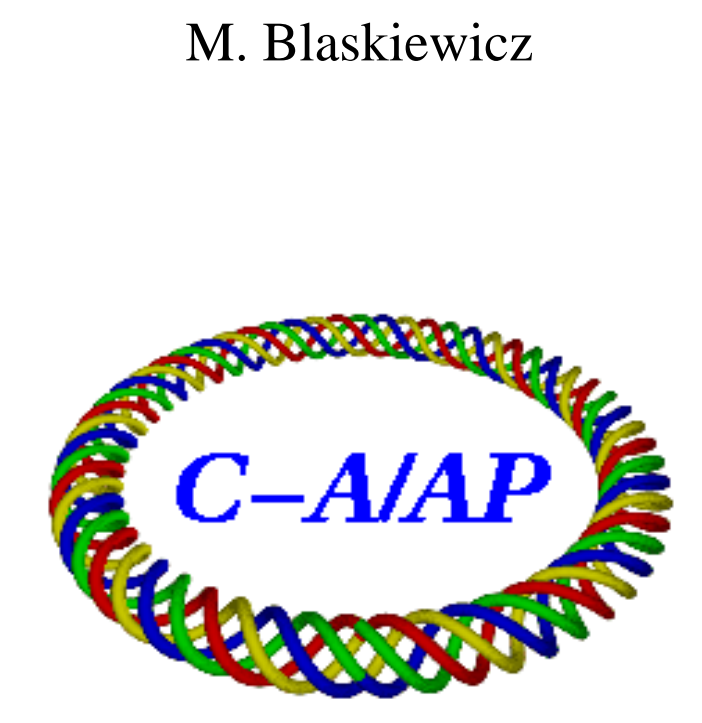

\section{Collider-Accelerator Department Brookhaven National Laboratory Upton, NY 11973}

Notice: This document has been authorized by employees of Brookhaven Science Associates, LLC under Contract No. DE-AC02-98CH10886 with the U.S. Department of Energy. The United States Government retains a non-exclusive, paid-up, irrevocable, world-wide license to publish or reproduce the published form of this document, or allow others to do so, for United States Government purposes. 


\title{
Copper coating specification for the RHIC arcs
}

\author{
M. Blaskiewicz* \\ $B N L$, Upton NY 11973, USA
}

\begin{abstract}
Copper coating specifications for the RHIC arcs are given. Various upgrade scenarios are considered and calculations of resistive wall losses in the arcs are used to constrain the necessary quality and surface thickness of a copper coating [1]. We find that $10 \mu \mathrm{m}$ of high purity copper will suffice.
\end{abstract}

\section{INTRODUCTION AND THEORY}

Typical parameters for an eRHIC [2] are shown in Table I. We will be concerned with ohmic losses on the pipe walls. For a round beam pipe the power loss per meter is given by [3]

$$
P^{\prime}=\frac{M Q^{2}}{4 \pi^{2} b T_{0}} \int_{0}^{\infty} d \omega R_{s}(\omega) e^{-\sigma_{s}^{2} \omega^{2} / c^{2}},
$$

where $R_{s}(\omega)$ is the surface resistivity as a function of angular frequency. For room temperature and low frequencies $R_{s}=\sqrt{Z_{0} \omega \rho / 2 c}$, where $Z_{0}=\mu_{0} c=377 \Omega$. Room temperature resistivities give ohmic losses of $0.5 \mathrm{~W} / \mathrm{m}$ for a stainless steel beam pipe and $0.12 \mathrm{~W} / \mathrm{m}$ for a copper one. The previously used upper limit for losses in the arcs is $0.5 \mathrm{~W} / \mathrm{m}$ [4]. Cooling the stainless steel pipe to $4 \mathrm{~K}$ will reduce it's resistivity but magneto-resistance will increase it. It therefore seems prudent to reduce the ohmic losses by coating the beam pipe with copper. A device for insitu coating is described in [1] and previous calculations have assumed that one can count on a factor of $R R R=100$ reduction in resistivity leading to a coating thickness of $5 \mu \mathrm{m}$. There are two problems with this procedure. Firstly, magneto-resistance at 3.45T and an RRR of 100 doubles the effective resistivity of the copper [5]. Secondly, even with an effective $R R R=50$, the mean free path of an electron in the copper is $\ell=2 \mu \mathrm{m}$, which is the skin depth at $20 \mathrm{MHz}$. This was estimated using the formula $[3] \ell=\left(\rho_{0} \ell_{0}\right) / \rho$ where $\rho_{0} \ell_{0}=6.6 \times 10^{-16} \Omega \mathrm{m}^{2}$ for copper. When the mean free path is longer than the skin depth the current at one location in the metal will be influenced by signficantly different electric fields at other locations and one needs to include convection in the electron transport [6]. This calculation was done in [6] for a ray of light with normal incidence. For wavenumber $k=\omega / c$ the electric field satisfies

$$
\frac{d^{2} E}{d r^{2}}+k^{2} E=\frac{-3 i Z_{0} k}{4 \rho \ell} \int_{0}^{d} d r_{1} E\left(r_{1}\right) k_{a}\left(\frac{r-r_{1}}{\ell}\right)
$$

TABLE I: RHIC ring parameters

\begin{tabular}{lc}
\hline \hline parameter & value \\
\hline circumference & $C=3834 \mathrm{~m}$ \\
revolution period and frequency & $T_{0}=12.8 \mu \mathrm{s}=1 / f_{0}$ \\
maximum charge per bunch & $Q=32 \mathrm{nC}$ \\
minimum rms bunch length & $\sigma_{s}=5 \mathrm{~cm}$ \\
bunches in the ring & $M=180$ \\
room temperature copper resistivity & $\rho_{c}=1.7 \times 10^{-8} \Omega \mathrm{m}[7]$ \\
RRR for copper & 100 \\
room temperature stainless resistivity & $\rho_{s}=3.6 \times 10^{-7} \Omega \mathrm{m}$ \\
beam pipe radius & $b=3.6 \mathrm{~cm}$ \\
\hline \hline
\end{tabular}

*Electronic address: blaskiewicz@bnl.gov 
where $r$ measures the depth of the material, $d$ is the thickness of the coating, and we have assumed diffuse reflection of the electrons at the interface as verified by measurements [8]. The time dependence is $\exp (-i \omega t)$ and the kernel function is

$$
k_{a}(x)=\int_{1}^{\infty} e^{-|x| s a}\left(\frac{1}{s}-\frac{1}{s^{3}}\right) d s,
$$

where $a=1-i \omega \ell / v_{\text {fermi }}$ and the Fermi velocity is $v_{f e r m i}=1.57 \times 10^{6} \mathrm{~m} / \mathrm{s}$ for copper. Note that

$$
\int_{-\infty}^{\infty} d x k_{a}(x)=4 / 3 a
$$

so that if the electric field is constant over many mean free paths equation (2) reduces to

$$
\frac{d^{2} E}{d r^{2}}+k^{2} E=-i \mu_{0} \omega J(r)
$$

with $J=\sigma E / a$ where $\sigma=1 / \rho$ is the low frequency conductivity. This result was in fact used to obtain the coefficient in (2).

The surface impedance is $Z_{s}=E / H$ where $H=B / \mu_{0}$ for our nonmagnetic materials and the fields are evaluated at the surface of the material. Faraday's law in cylindrical coordinates gives

$$
\frac{\partial E_{r}}{\partial z}-\frac{\partial E_{z}}{\partial r}=i \omega \mu_{0} H_{\phi}
$$

For beam generated fields with $J=\sigma E$ in the wall of a cylindrical pipe, the second term on the left is much bigger than the first [9] giving

$$
Z_{s} \approx-i k Z_{0} \frac{E_{z}}{E_{z}^{\prime}}
$$

where $E_{z}^{\prime}=\partial E_{z} / \partial r$ evaluated at the surface and $R e\left(Z_{s}\right)=R_{s}(\omega)$ in equation (1). This is the same result one gets for the reflection of normally incident light which allows us to use the same expressions for surface impedance in both cases. For $d \rightarrow \infty$ equation (2) can be solved in closed form using Laplace transforms [10] and expressions for the surface impedance may be obtained. One finds

$$
\frac{E_{z}^{\prime}}{E_{z}}=-\frac{a}{\pi \ell} \int_{0}^{\infty} \ln \left\{1+\frac{\xi \chi(t)}{t^{2}}\right\} d t
$$

where the fields are evaluated at the surface of the metal, $\xi=3 \ell^{2} / 2 \delta^{2} a^{3}, \delta=\sqrt{2 \rho / k Z_{0}}$ is the skin depth and $\chi(t)=2 t^{-3}\left[\left(1+t^{2}\right) \arctan (t)-t\right]=4 / 3+4 t^{2} / 15+O\left(t^{4}\right)$. While equation (2) holds for diffuse reflection of electrons of the metal surface it is possible to solve the kinetic equations assuming electrons undergo perfect reflection at the interface. In this case

$$
\frac{E_{z}}{E_{z}^{\prime}}=-\frac{2 \ell}{\pi a} \int_{0}^{\infty} \frac{d t}{t^{2}+\xi \chi(t)}
$$

In equations (4) and (5) $\mathrm{r}$ is zero at the interface between vacuum and copper and increases with depth in the copper. This produces a sign change in the derivatives which is simple but needs to be looked after.

When the coating thickness is finite one may solve equation(2) numerically. To do this first take the dimensionless variable $u=r / \ell$. Next we take $u=0$ to be at the interface between the copper and the stainless steel. Equation (2) becomes

$$
\frac{d^{2} E}{d u^{2}}+k^{2} \ell^{2} E=-i A \int_{0}^{d / \ell} d u_{1} E\left(u_{1}\right) k_{a}\left(u-u_{1}\right)
$$


where $A=3 Z_{0} k \ell^{2} / 4 \rho$. Inside the stainless for $u<0$ one has $E(u)=E_{0} \exp \left[(1-i) u \ell / \delta_{s s}\right]$ where $\delta_{s s}$ is the skin depth in the stainless and $E_{0}$ is a constant. Since $E_{z}$ and $H_{\phi}$ are continuous at the boundary one has $E(u)=$ $E_{0}+u E_{0}(1-i) \ell / \delta_{s s}+O\left(u^{2}\right)$ for small positive $u$ inside the copper. Integrating equation (6) twice with respect to u gives

$$
E(u)+\int_{0}^{u}\left(u-u_{2}\right) d u_{2}\left(k^{2} \ell^{2} E\left(u_{2}\right)+i A \int_{0}^{d / \ell} d u_{1} E\left(u_{1}\right) k_{a}\left(u_{2}-u_{1}\right)\right)=E_{0}+u E_{0}^{\prime},
$$

where $E_{0}^{\prime}=E_{0}(1-i) \ell / \delta_{s s}$. To evaluate the surface impedance for thick copper coatings equations (4) and (5) may be evaluated numerically. To understand the effect of coating thickness we need to solve equation (7) numerically. To do this set $d / \ell=\hat{d}$ and divide the interval $[0, \hat{d}]$ into $K$ equal segments of length $\Delta=\hat{d} / K$. Let $E_{m}=E\left(u_{m}\right)$ be the field at $u_{m}=[m-1 / 2] \Delta$ for $m=1,2, \ldots K$. The term in (7) proportional to $k^{2} \ell^{2}$ is extremely small and will be dropped leaving

$$
E_{n}+i A \Delta^{3} \sum_{j=1}^{K} E_{j} \sum_{m=1}^{n}(n-m) \hat{k}_{a}(m-j)=E_{0}+E_{0}^{\prime}(n-1 / 2) \Delta .
$$

where we have defined a smoothed version of $k_{a}(x)$ namely

$$
\hat{k}_{a}(x)=\frac{1}{\Delta} \int_{x-\Delta / 2}^{x+\Delta / 2} d x_{1} k_{a}\left(x_{1}\right)
$$

The smoothing makes $\hat{k}_{a}(0)$ finite and equation (8) can now be solved via matrix inversion.

Before closing this section we note that a quantum mechanical theory of the anomalous skin effect exists [11]. This theory produces the smoothing function $\exp (-|x| / \ell)$. For large $r, k_{a}(r / \ell)=2 \exp (-a|r| / \ell) /(a|r| / \ell)^{2}$

\section{RESULTS}

Figures 1 through 4 show solutions of equation (8) for two frequencies. The value of $R R R=50$ used in the calculation corresponds to $3.45 \mathrm{~T}$. For these figures the electric field is set to one at $u=0$. From Figure 1 it is seen that the electric field at $10 \mathrm{MHz}$ has a magnitude greater than 10 at the surface of the metal. Therefore, the effective surface current at the stainless steel is less than $1 / 10$ th the surface current at the pipe wall and the dissipation in the stainless steel is less than $1 \%$ of what it would be without copper coating. For the high frequency case the field in the stainless is much smaller. Thsi plot is included to show that $k_{a}$ begins to have an imaginary part only at very high frequencies.

Figures 5 and 6 show surface impedance calculations using equations (4) and (8) for $R R R=50$ and $R R R=25$ respectively. Using equation (1) and the beam parameters in Table 1, the power per unit frequency for the two values of $\mathrm{RRR}$ are shown in Figure 7. For $R R R=50$ at $3.45 \mathrm{~T}$ the power per unit frequency is $0.064 \mathrm{Watts} / \mathrm{meter}$. For $R R R=25$ at $3.45 \mathrm{~T}$ the power per unit frequency is 0.079 Watts/meter. Both the values are well below the $0.5 \mathrm{Watt} /$ meter) limit considered in [4]. For a fixed bunch length of $\sigma_{s}=5 \mathrm{~cm}$ the results are easily extended to different numbers of bunches and charges per bunch. For instance, with $1.0 \times 10^{11}$ protons per bunch the charge per bunch is $16 \mathrm{nC}$. For 2000 such bunches and $R R R=50$ the loss rate is $P=(1 / 2)^{2}(2000 / 180) 0.064$ Watts $/$ meter $=$ 0.18 Watts/meter which is still acceptable.

\section{Acknowledgements}

Thanks to Wolfram Fischer for useful discussions and encouragement. Slides provided by Elias Metral were very helpful.

[1] A. Hershcovitch, M. Blaskiewicz, J.M. Brennan, W. Fischer, C-J Liaw, W. Meng, A. Custer, M. Erickson, N. Jamshidi, H.J. Poole, IPAC10, p1509, tupea082, (2010). 


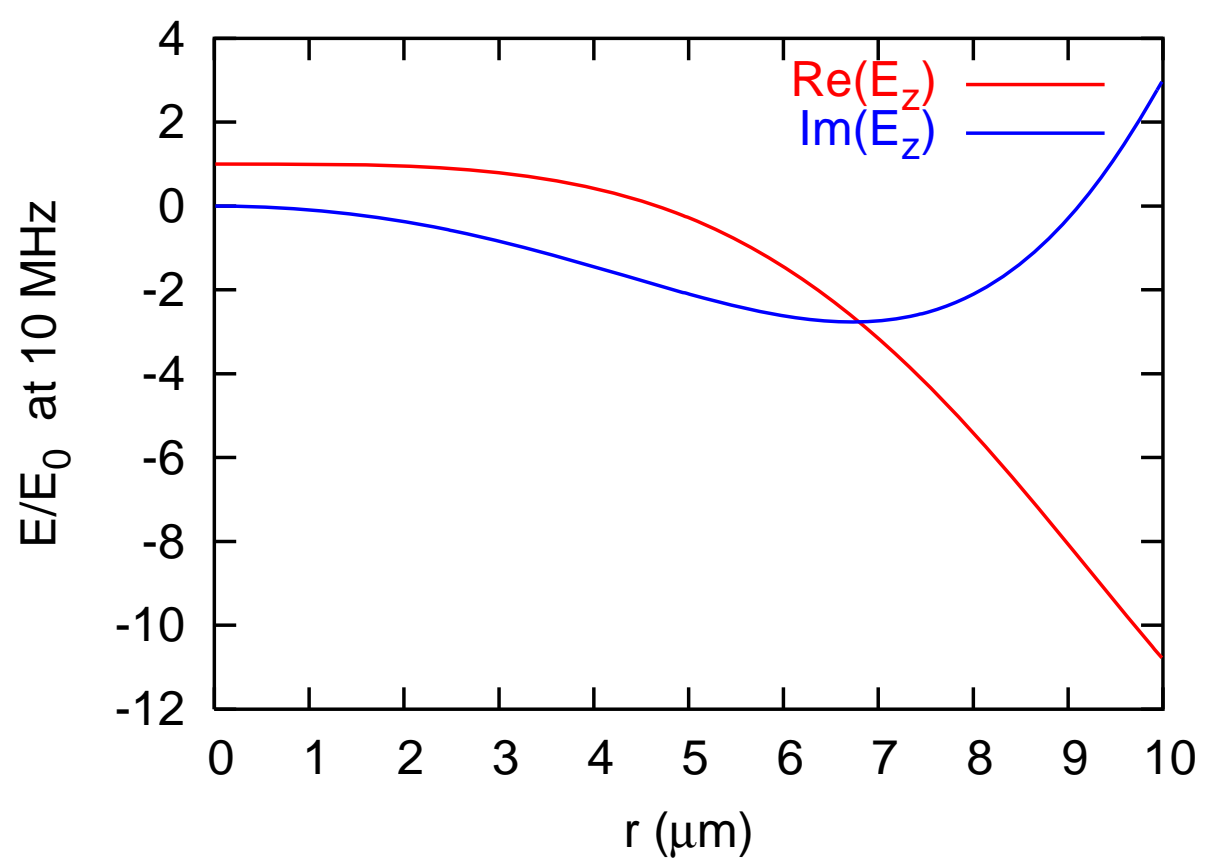

FIG. 1: Electric field with stainless steel for $r<0$ and copper with $R R R=50$ for $0<r<10 \mu$ m. The frequency is 10 MHz.

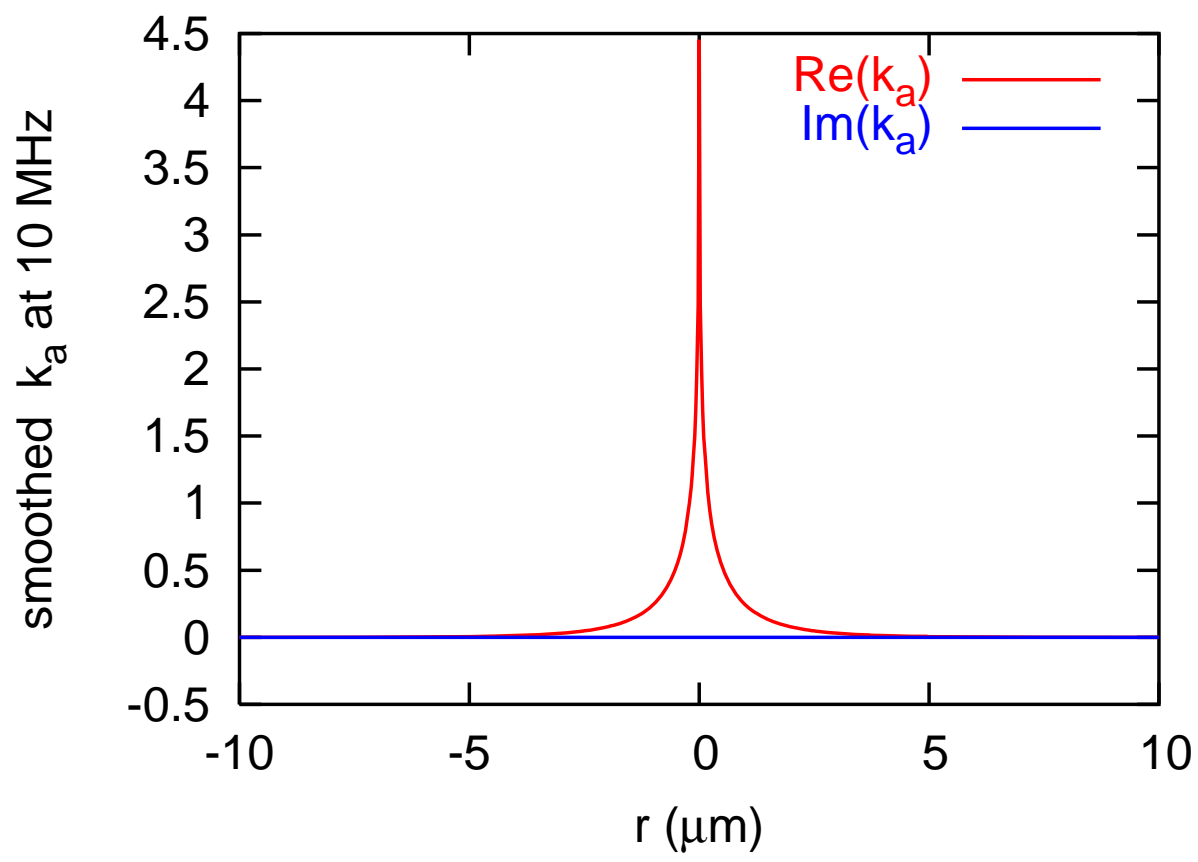

FIG. 2: The function $\hat{k}_{a}(r / \ell)$ for the parameters in Figure 1.

[2] V. Litvinenko wexmh02 IPAC10 (2010).

[3] W. Chou, F. Ruggiero, LHC Project Note 2 (SL/AP) (1995).

[4] A.G. Ruggiero, S. Peggs RHIC/AP/46 (1994).

[5] F. Caspers et. al. LHC Project report \# 307, (1999).

[6] G.E.H Reuter and E.H. Sondheimer Proc. R. Soc. London A 195 p336 (1948).

[7] CRC Handbook of chemistry and physics. section 12-232.

[8] R.G. Chambers Proc. R. Soc. London A 215 p481 (1952).

[9] Chao's book 


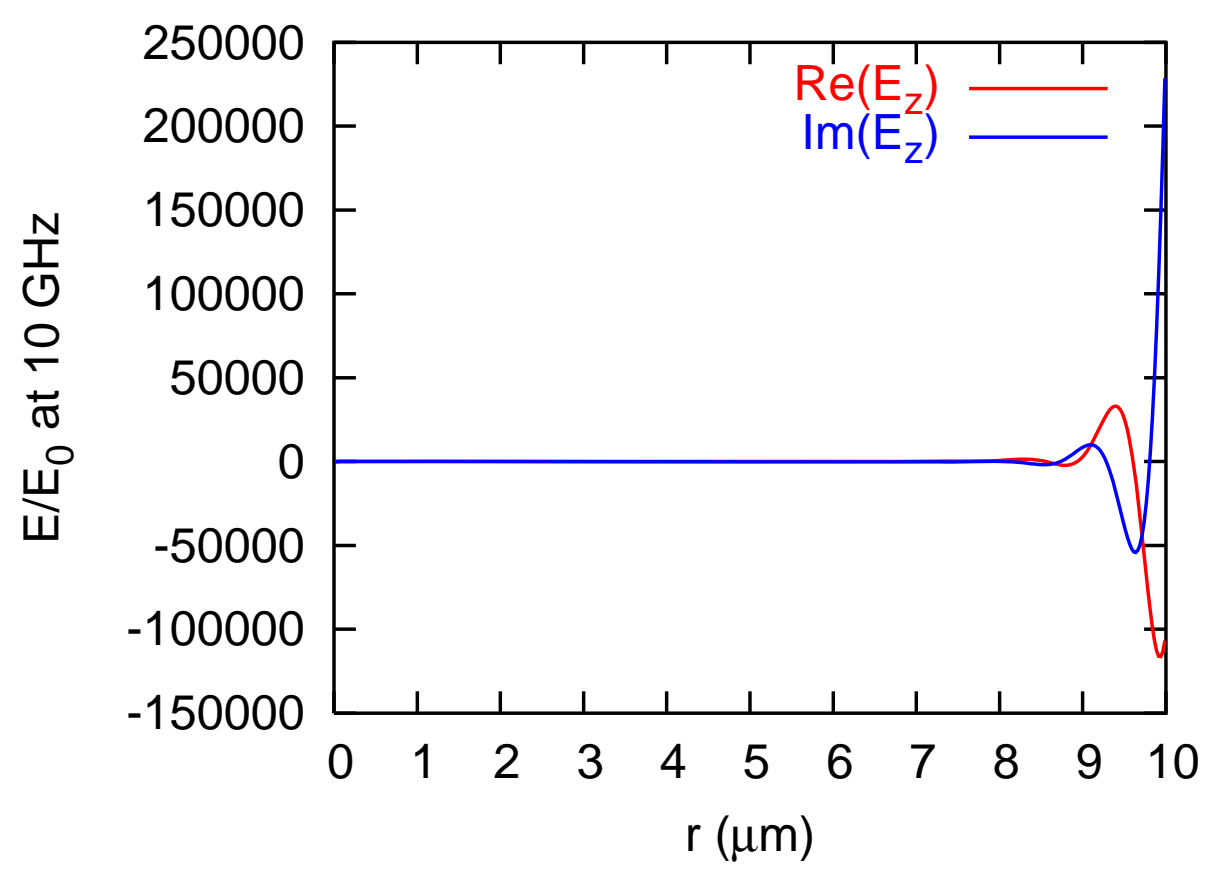

FIG. 3: Electric field with stainless steel for $r<0$ and copper with $R R R=50$ for $0<r<10 \mu$ m. The frequency is 10 GHz.

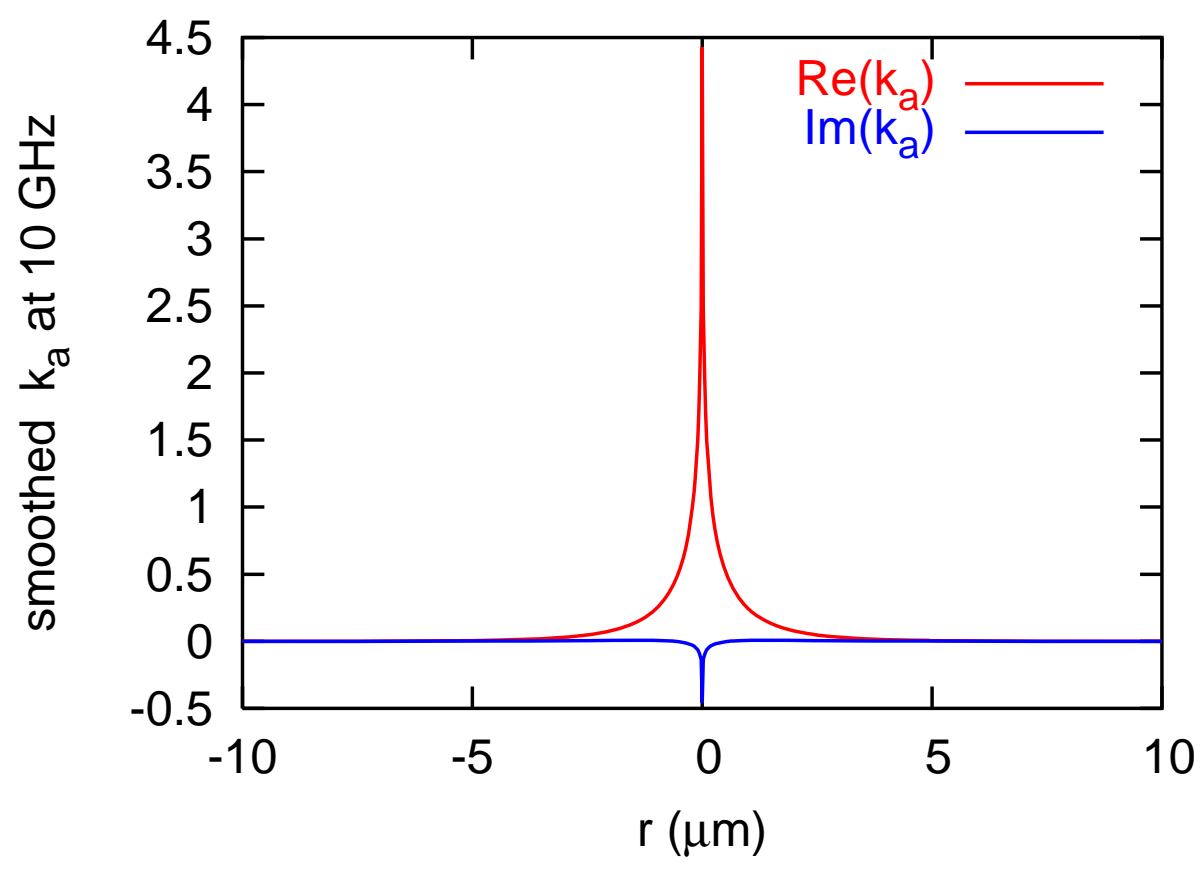

FIG. 4: The function $\hat{k}_{a}(r / \ell)$ for the parameters in Figure 3.

[10] R.B. Dingle Physica 19 p311 (1953).

[11] D.C. Mattis and J. Bardeen Phys. Rev. 111 \# 2 p412 (1958). 


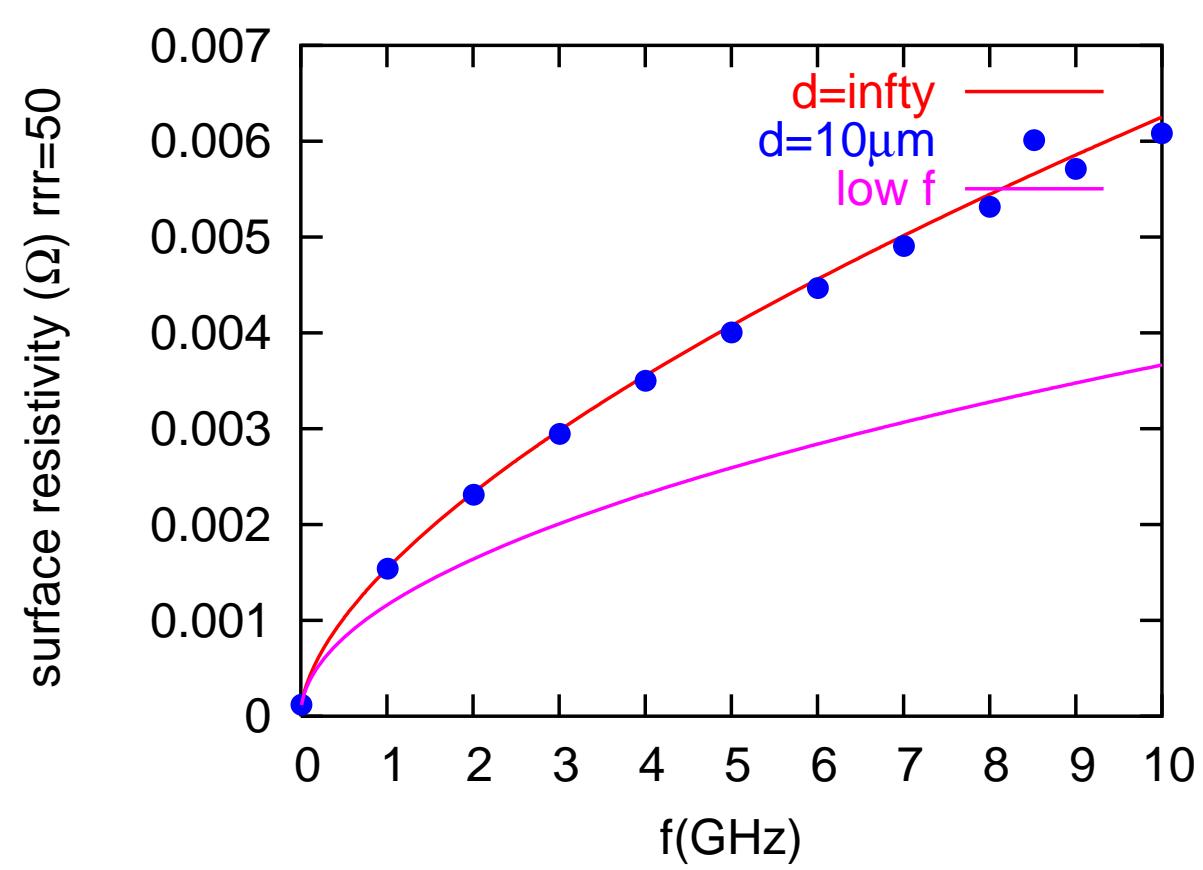

FIG. 5: Surface resistance calculations for $R R R=50$ with $10 \mu \mathrm{m}$ of copper using (8) are displayed with blue dots. The red line used equation (4).

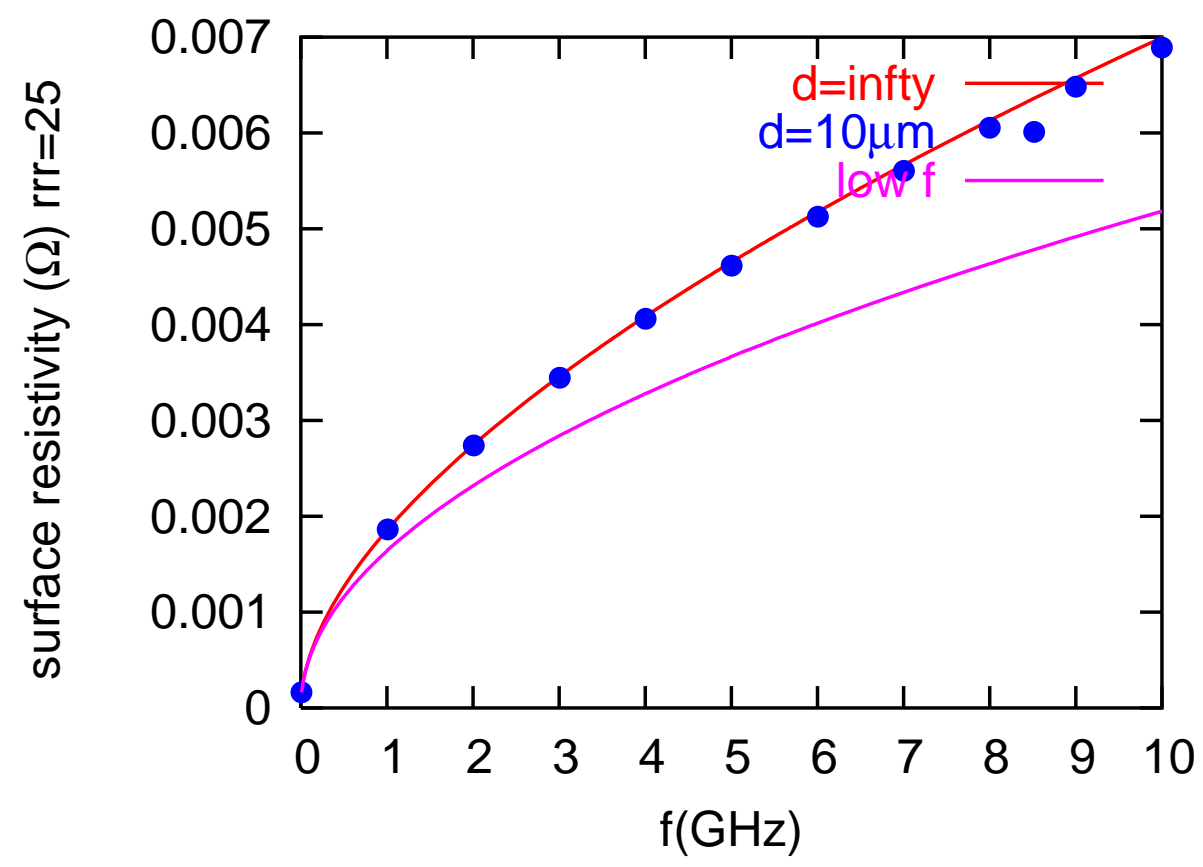

FIG. 6: Surface resistance calculations for $R R R=25$ with $10 \mu \mathrm{m}$ of copper using (8) are displayed with blue dots. The red line used equation (4) . 


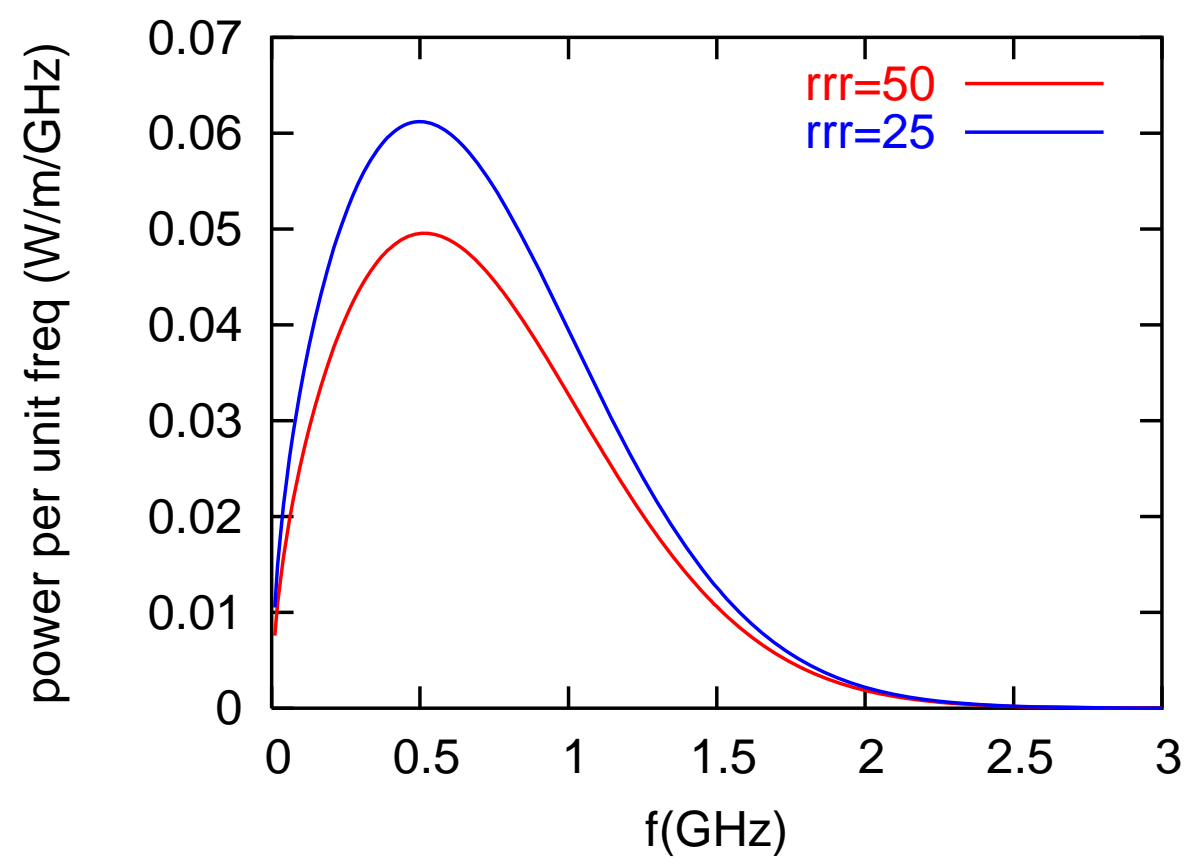

FIG. 7: Ohmic loss per unit length per unit frequency using equation (4) and (1). For $R R R=50$ the integral is 0.064 Watts $/ \mathrm{m}$. For $R R R=25$ the integral is 0.079 Watts $/ \mathrm{m}$. 